\title{
The study of trunk and pelvic movement on mediolateral ramps of various ramp angles during one-legged standing
}

\author{
Sang-Yeol Lee', Se-Yeon Park ${ }^{2 * *}$ \\ 'Department of Physical Therapy, College of Science, Kyungsung University, Korea \\ ${ }^{2}$ Department of Physical Therapy, College of Health Science, Kaya University, Korea
}

The purpose of this study was to identify the instability of the trunk and the pelvis of subjects who were standing on differently angled ramps under conditions in which the ramps were crossed. Changes in the trunk and the pelvis while each subject was standing on one leg in the direction of the cross ramps at four angles $\left(0^{\circ}, 5^{\circ}, 10^{\circ}\right.$, and $\left.15^{\circ}\right)$ were measured. To identify instability, the trunk inclination, trunk imbalance, pelvic position, pelvic rotation, and pelvic torsion of 20 healthy male subjects were measured. The measurement was conducted using a three-dimensional spinal diagnostic imaging system (Back Mapper). According to the results of the measurement, trunk imbalance, pelvic position, pelvic rotation, and pelvic torsion differed between the medial and lateral ramps and with different ramp angles. This study's results suggested that different mediolateral ramp conditions altered the pelvic and trunk alignment. With the $15^{\circ}$ ramp, the anterior tilt and lateral tilt of the pelvis increased regardless of ramp direction. The $10^{\circ}$ ramp condition altered the pelvic rotation to the contralateral side. In addition, the lateral trunk tilt was elevated with the lateral down foot condition.

Keywords: Imbalance, Mediolateral ramp, Trunk movement

\section{INTRODUCTION}

Pedestrian environments are influenced by natural environmental conditions and physical environmental conditions, and the influence of these conditions has both direct and indirect effects on walking. As one example of these environments, inclined roads have been developed to function as moving paths to overcome height differences and to facilitate walking. Recently, pursuant to the Act on guarantee of promotion of convenience of persons with disabilities, the aged, pregnant women, etc., to improve the safety and accessibility of the vulnerable who experience inconvenience while moving, standards to facilitate vertical movements in spaces with height differences have been developed (Korea Legislation Research Institute, 2015). Meanwhile, due to the urban concentration of the population due to economic growth and the prevalence of poor hillside villages that were formed as refugees settled after the Korean War, the number of people living in residential spaces

with high altitudes has increased. However, since such residential spaces were not protected by the Act on guarantee of promotion of convenience of persons with disabilities, the aged, pregnant women, etc., these areas developed inclined roads. Because of the prevalence of these types of roads in these areas, people living in these areas are frequently exposed to environments where they cross inclined roads horizontally instead of ascending or descending.

Walking on a ramp requires involves effort from the muscle group of the lower limbs, such as the gluteus maximus, hamstring muscle, and quadriceps muscle, to produce higher levels of thrust than that which is required for walking on general flatlands and for speed control (Lay et al., 2006; Lay et al., 2007), and walking on a ramp requires body strategies for stable support (Lange et al., 1996; Lay et al., 2006). However, most studies related to walking on a ramp have been limited to ascending/descending ramps rather than walking horizontally across the ramps (Han et al., 2009). Recently, a study was conducted on the characteristics of the mus-

${ }^{\star}$ Corresponding author: Se-Yeon Park (D) https://orcid.org/0000-0002-5769-8732 Department of Physical Therapy, Kaya University, 208 Samgye-ro, Gimhae 50830, Korea

Tel: +82-55-344-5295, Fax: +82-55-344-5285, E-mail: arclain@naver.com

Received: June 22, 2017 / Accepted: August 10, 2017 
cles that control the ankle joint and the hip joint when ramps were crossed. According to the results of this study, the use of the gluteus medius muscle increased significantly when people were crossing ramps with a $15^{\circ}$ angle (Kim and Lee, 2017), indicating that the role of the gluteus medius muscle is more important during walking and one-legged standing on inclined roads to control the movements of the trunk and the lower limbs in order maintain posture (Finch et al., 1991). In addition, another study reported that the use of the peroneus longus muscle significantly increased when $10^{\circ}$ ramps were crossed. This means that on $10^{\circ}$ ramps, body stability is controlled by ankle joint movements (Lee et al., 2017).

In general, balance ability means the ability to maintain one's center of mass on basal planes (Shumway-Cook and Woollacott, 2007). To maintain balance, human bodies appropriately use ankle strategies, hip strategies, and strategies that combine both ankle and hip strategies (Horak and Nashner, 1986). The use of such strategies should be accompanied by the contraction of surrounding muscles (King et al., 2012). However, in the elderly, the contraction of such muscles is sometimes not accompanied by the contraction of surrounding muscles or their response time is delayed, which both increase the risk of falls (King et al., 2012).

Although previous studies have examined muscle control while crossing medial/lateral slopes, these studies have not directly presented changes in the trunk and the pelvis in these conditions. Therefore, in this study, changes in the trunk and the pelvis according to the medial/lateral slopes of ramps will be analyzed assuming ramp crossing to provide basic data on the most appropriate angles of inclination for walker safety.

\section{MATERIALS AND METHODS}

\section{Study population}

Twenty asymptomatic males were recruited from a local university using convenience sampling. The subjects had a mean \pm standard deviation age of 22.84 2.24 years (range, 20-26 years). Subjects with a history of musculoskeletal problems, such as back pain or lower extremity pain, in the past 6 months were excluded. The study population consisted of 20 males, who had a height and weight of $172.26 \pm 4.20 \mathrm{~cm}$ and $64.20 \pm 8.20 \mathrm{~kg}$, respectively. The average body mass index was $21.69 \pm 2.23 \mathrm{~kg} / \mathrm{m}^{2}$. Ethics approval for this study was obtained from the Kyeongsung University Faculty of Health Sciences Human Ethics Committee (KSU-17-04-004). The subjects were informed of the study's purpose and content prior to the study.

\section{Instrumentation}

The present study investigated spinal alignment according to medial and lateral ramp angles using a three-dimensional spinal diagnostic imaging system (Back Mapper, ABW, Frickenhausen, Germany). This three-dimensional spinal diagnostic imaging system could perform multivariate analysis and has been identified as a valid method for a spinal alignment assessment (Drerup and Hierholzer, 1987; Drerup and Hierholzer, 1994). The device assessed the variables in the frontal plane (trunk imbalance, pelvic position), the sagittal plane (trunk inclination, pelvic torsion), and the horizontal plane (pelvic rotation). Trunk imbalance represented the trunk's lateral tilt angle against the vertical line. Pelvic position represented pelvic lateral tilt angle in the frontal plane. Trunk inclination represented trunk tilt in the sagittal plane. Pelvic torsion angle represented the degree of anterior and posterior tilt of the pelvis. For the rotation of the pelvis on the horizontal plane, a positive value indicated rotation to right side, and a negative value indicated rotation to left side (Cho, 2016; Yi et al., 2016).

\section{Experimental procedure}

The subjects were measured by exposing their upper extremity and pelvic area and were asked to stand with their feet spread apart at shoulder width. Any reflective materials, such as necklaces or earrings, were removed to minimize measurement errors. With the subject's dorsal side towards the camera, measurements were performed with each subject taking a neutral double stance and a one-leg stance while standing on medial and lateral ramps with angles of $5^{\circ}, 10^{\circ}$, and $15^{\circ}$ (Fig. 1). Marking the position of the feet ensured the reproducibility of the foot positions. The examiner manually placed reflective markers on $\mathrm{C} 7$, on both sides of the scapular inferior angle, on the bilateral posterior superior iliac spine, and on the center of the sacrum, totaling six reflective markers placed on each subject (Yi et al., 2016). Prior to measurement, each participant was instructed verbally and visually how to perform a one-leg stance on the medial and lateral ramps, and each participant was allowed three minutes of practice time to be-

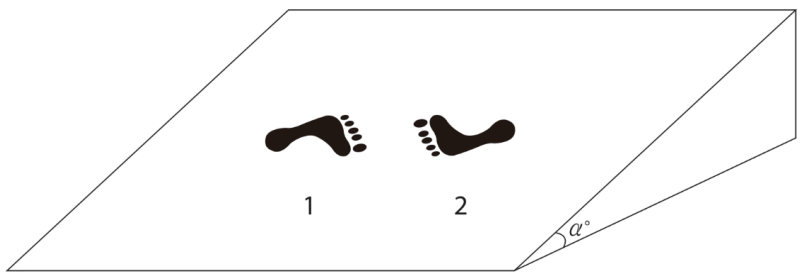

Fig. 1. The experimental conditions of this study. 1, foot on medial ramp; 2 , foot on lateral ramp. 
Table 1. Comparison of trunk and pelvic parameters with different ramp conditions during one-legged standing $(\mathrm{n}=20)$

\begin{tabular}{|c|c|c|c|c|c|c|c|c|c|c|}
\hline \multirow{2}{*}{ Ramp condition } & \multirow{2}{*}{$\begin{array}{c}0^{\circ} \text { (double } \\
\text { stance) }\end{array}$} & \multirow{2}{*}{$\begin{array}{c}0^{\circ} \text { (one-leg } \\
\text { stance) }\end{array}$} & \multicolumn{3}{|c|}{ Foot on lateral ramp } & \multicolumn{3}{|c|}{ Foot on medial ramp } & \multirow{2}{*}{ F-value } & \multirow{2}{*}{$P$-value } \\
\hline & & & $5^{\circ}$ & $10^{\circ}$ & $15^{\circ}$ & $5^{\circ}$ & $10^{\circ}$ & $15^{\circ}$ & & \\
\hline Trunk inclination $\left({ }^{\circ}\right)$ & $-1.20 \pm 1.76$ & $-2.10 \pm 2.83$ & $-2.35 \pm 3.50$ & $-1.90 \pm 3.04$ & $-2.80 \pm 3.00$ & $-2.85 \pm 3.60$ & $-2.80 \pm 3.93$ & $-3.55 \pm 3.93$ & 1.94 & 0.14 \\
\hline Trunk imbalance $\left({ }^{\circ}\right)$ & $0.05 \pm 1.23$ & $1.10 \pm 2.49$ & $2.05^{\dagger} \pm 2.16$ & $2.70^{\dagger} \pm 2.20$ & $2.75^{\dagger} \pm 2.40$ & $1.30 \pm 2.30$ & $1.20 \pm 1.82$ & $1.70 \pm 2.79$ & 5.28 & $0.01^{*}$ \\
\hline Pelvic position $\left({ }^{\circ}\right)$ & $1.25 \pm 1.07$ & $2.35 \pm 1.81$ & $2.90 \pm 2.17$ & $3.75^{\dagger} \pm 2.79$ & $3.50^{\dagger} \pm 2.24$ & $3.40 \pm 3.33$ & $3.55^{\dagger} \pm 2.16$ & $4.45^{\dagger} \pm 3.47$ & 7.03 & $0.00^{*}$ \\
\hline Pelvic rotation $\left({ }^{\circ}\right)$ & $-0.60 \pm 2.30$ & $-2.45 \pm 3.49$ & $-5.40^{\dagger} \pm 4.31$ & $-5.10^{\dagger} \pm 4.01$ & $-4.60^{\dagger} \pm 5.02$ & $-3.20 \pm 4.12$ & $-5.35^{\dagger} \pm 4.97$ & $-5.50^{\dagger} \pm 5.37$ & 3.96 & $0.01^{*}$ \\
\hline Pelvic torsion $\left({ }^{\circ}\right)$ & $-0.10 \pm 4,04$ & $3.60 \pm 5.81$ & $5.70^{\dagger} \pm 5.77$ & $6.20^{\dagger} \pm 5.54$ & $5.45^{\dagger} \pm 6.55$ & $3.40 \pm 5.99$ & $3.60 \pm 5.86$ & $5.20^{\dagger} \pm 5.64$ & 2.76 & $0.05^{*}$ \\
\hline
\end{tabular}

Values are presented as mean \pm standard deviation.

${ }^{*} P<0.05$, statistically significant. ${ }^{~}$ Significantly different to values of double stance $(P<0.05)$ according to Sidak's measure.

come familiar with the ramps. Each participant had $30 \mathrm{sec}$ of rest between each ramp condition. Measurement during the one-leg stance was conducted on each subject's dominant side.

\section{Statistical analysis}

PASW Statistics ver. 18.0 (SPSS Inc., Chicago, IL, USA) was used to determine the significance of the differences in the spinal alignment values of each ramp condition. A one-way repeated measures analysis of variance was used to determine the effects of each ramp condition on the spinal alignment data. In all analyses, $P<0.05$ was taken to indicate statistical significance.

\section{RESULTS}

There were significant differences in trunk imbalance, pelvic position, pelvic rotation, and pelvic torsion angles according to mediolateral ramp angle (Table 1) $(P<0.05)$. Trunk imbalance, which represented trunk tilt against the vertical line, was significantly higher with medial ramp angles of $5^{\circ}, 10^{\circ}$, and $15^{\circ}$ than with the trunk imbalance angle during the double stance posture $(P<0.05)$.

Pelvic position, which represented the lateral tilt of the pelvis, showed significantly higher values with both medial and lateral ramp angles of $10^{\circ}$ and $15^{\circ}$ than with the lateral tilt of the pelvis in the double stance posture $(P<0.05)$.

The pelvic rotation angles of $5^{\circ}, 10^{\circ}$, and $15^{\circ}$ on the medial ramp and $10^{\circ}$ and $15^{\circ}$ on the lateral ramp were significantly lower than that of the double stance posture $(P<0.05)$. Lastly, the $5^{\circ}$, $10^{\circ}$, and $15^{\circ}$ medial ramps and $15^{\circ}$ lateral ramp increased the pelvic torsion angle significantly more than the double stance posture $(P<0.05)$.

\section{DISCUSSION}

The present study has investigated the effects of different mediolateral ramp conditions on trunk and pelvic alignment during a one-leg stance by comparing trunk and pelvic alignment during this stance with the trunk and pelvic alignment during a double stance. This study used mediolateral ramp conditions that included $5^{\circ}, 10^{\circ}$, and $15^{\circ}$ slopes. In a perspective of limb length discrepancy, $1 \mathrm{~cm}$ of discrepancy was shown to generate tilted trunk and pelvic alignment (Khamis and Carmeli, 2017). The 5 $5^{\circ}, 10^{\circ}$, and $15^{\circ}$ slopes used in the present study can be converted to tangent values of $0.087,0.176$, and 0.267 , respectively. Using a simple calculation, these values mean that the $5^{\circ}$ slopes could generate $1 \mathrm{~cm}$ of limb length discrepancy when the width of the slopes was over $12 \mathrm{~cm}$. For an asymptomatic and healthy population, this discrepancy cannot affect balance ability, but this discrepancy elevates the risk of falling and musculoskeletal problems for an elderly and symptomatic population (Lee et al., 2017). However, only a few studies have investigated the effects of slope angles on pelvic and trunk alignment.

The results of present study showed that trunk imbalance that manifested in lateral trunk tilt was significantly higher with the $5^{\circ}, 10^{\circ}$, and $15^{\circ}$ lateral down foot conditions than with the double stance conditions. The mean differences between the lateral down foot conditions and the double stance ranged from $2^{\circ}$ to $4^{\circ}$. In a classical biomechanical study of gait, the average range of trunk tilt was approximately $2^{\circ}$ during the dynamic gait condition (Sartor et al., 1999). Considering that the present study investigated lateral trunk tilt during a static one-leg stance on a slope, the degree to which tilt could be generated during a dynamic gait on slope conditions and this tilt's effects on falling risk for the elderly are unclear.

Lateral down foot conditions force the ankle joint into a supinated position that includes ankle plantar flexion and adduction (Neumann, 2002). Although the present study did not investigate center of gravity and the ground reaction force, which prevented us from affirming the force direction, the generated force might be in the cranial and lateral direction against the supinated ankle joint. Matching the line of the ground reaction force to the 
center of mass in our body is an adaptation that is essential for maintaining balance ability. The elevated lateral tilt on the lateral down foot condition might be caused from this adaptation.

The results of the present study showed significant differences in pelvic alignment variables between double stance and mediolateral ramp conditions. Pelvic torsion, which represented the anterior tilt of the pelvis, and pelvic position, which represented the pelvic lateral tilt, were increased. Although the present study did not investigate the pathologic condition, changes in the lower limb condition reportedly alter the pelvic and spinal alignments. A recent study demonstrated that subjects with patellofemoral osteoarthritis had greater pelvic anterior tilt and lateral tilt during the stance phase of walking (Crossley et al., 2017). A classical geriatric study also demonstrated that body sway increased and lateral stability control decreased with increases in age (Maki and Mcllroy, 1996). Considering the present study's results of the $15^{\circ}$ lateral and medial down foot conditions, balance dysfunction could occur for the elderly, leading to falls and injuries regardless of slope direction.

Pelvic rotation was only represented by negative values in present study, which manifested in contralateral rotation against the stance limb. On the $10^{\circ} \mathrm{ramp}$, the pelvis was rotated to the contralateral limb regardless of ramp direction. Although we thought the present result of pelvic rotation was measured within the static one-leg stance, increased rotation to contralateral direction can reduce the efficacy of the gait during walking. Pelvic rotation has been described as an essential element of forward progression during gait (Neumann, 2002). However, increased pelvic rotation in the contralateral direction could alter the forward progression of the pelvis.

The present study examined the effects of various ramp angles on pelvic and trunk alignment. The results highlighted the risks of these ramp conditions; however, several of the present study's limitations should be addressed by future studies. The most important limitation was that the spinal alignment data was collected only in the static phase and not the dynamic walking phase. Second, we could not record kinetic data, such as ground reaction force, throughout the experiment, so the explanation of center of mass could not be supported. The third limitation was the exclusion of sagittal kinematic values because the previous study reported problems measuring sagittal kinematics with video rasterstereography (Schroeder et al., 2015).

This study suggested that the mediolateral ramp condition altered pelvic and trunk alignment. On a $15^{\circ}$ ramp, the anterior tilt and lateral tilt of the pelvis increased regardless of ramp direction.
The $10^{\circ}$ ramp condition could alter pelvic rotation to the contralateral side. In addition, lateral trunk tilt was elevated with a lateral down foot condition.

\section{CONFLICT OF INTEREST}

No potential conflict of interest relevant to this article was reported.

\section{ACKNOWLEDGMENTS}

This work was supported by the National Research Foundation of Korea (NRF) grant funded by the Korea government (MSIP; Ministry of Science, ICT \& Future Planning) (No. 2017R1C1B 5015093).

\section{REFERENCES}

Cho M. Effects of pelvic adjustment on pelvic posture and angles of the lower limb joints during walking in female university students. J Phys Ther Sci 2016;28:1284-1288.

Crossley KM, Schache AG, Ozturk H, Lentzos J, Munanto M, Pandy MG. People with patellofemoral OA walk with different pelvic and hip kinematics compared to healthy aged-matched controls. Arthritis Care Res (Hoboken) 2017 Apr 24 [Epub]. https://doi.org/10.1002/acr.23261.

Drerup B, Hierholzer E. Back shape measurement using video rasterstereography and three-dimensional reconstruction of spinal shape. Clin Biomech (Bristol, Avon) 1994;9:28-36.

Drerup B, Hierholzer E. Movement of the human pelvis and displacement of related anatomical landmarks on the body surface. J Biomech 1987;20:971-977.

Finch L, Barbeau H, Arsenault B. Influence of body weight support on normal human gait: development of a gait retraining strategy. Phys Ther 1991;71:842-855.

Han JT, Gong WT, Lee YS. Comparison of muscle activity with lower extremity during stairs and ramp climbing of old adults by EMG. J Korean Soc Phys Ther 2009;21:35-40.

Horak FB, Nashner LM. Central programming of postural movements: adaptation to altered support-surface configurations. J Neurophysiol 1986;55:1369-1381.

Khamis S, Carmeli E. Relationship and significance of gait deviations associated with limb length discrepancy: a systematic review. Gait Posture 2017;57:115-123.

Kim SC, Lee SY. Gluteus medius muscle activities according to various angle of mediolateral ramp during cross walking and one-leg stand- 
ing. J Korean Soc Phys Med 2017;12:53-57.

King GW, Stylianou AP, Kluding PM, Jernigan SD, Luchies CW. Effects of age and localized muscle fatigue on ankle plantar flexor torque development. J Geriatr Phys Ther 2012;35:8-14.

Korea Legislation Research Institute. Act on guarantee of promotion of convenience of persons with disabilities, the aged, pregnant women, etc. [Internet]. Seoul: Ministry for Health, Welfare and Family Affairs; 2015 [cited 2015 Jan 28]. Available from: https:/elaw.klri.re.kr/kor_service/lawView.do?lang=ENG\&hseq=37706.

Lange GW, Hintermeister RA, Schlegel T, Dillman CJ, Steadman JR. Electromyographic and kinematic analysis of graded treadmill walking and the implications for knee rehabilitation. J Orthop Sports Phys Ther 1996;23:294-301.

Lay AN, Hass CJ, Gregor RJ. The effects of sloped surfaces on locomotion: a kinematic and kinetic analysis. J Biomech 2006;39:1621-1628.

Lay AN, Hass CJ, Richard Nichols T, Gregor RJ. The effects of sloped surfaces on locomotion: an electromyographic analysis. J Biomech 2007; 40:1276-1285.

Lee SY, Lee SM, Jung JM. Peroneus Longus activity according to various angles of a ramp during cross-ramp walking and one-legged standing. J Back Musculoskelet Rehabil 2017 Jun 3 [Epub]. https:/doi.org/ 10.3233/BMR-150510.

Maki BE, Mcllroy WE. Postural control in the older adult. Clin Geriatr Med 1996;12:635-658.

Neumann DA. Kinesiology of the musculoskeletal system: foundations for physical rehabilitation. St Louis: Mosby; 2002.

Sartor C, Alderink G, Greenwald H, Elders L. Critical kinematic events occurring in the trunk during walking. Hum Mov Sci 1999;18:669-679.

Schroeder J, Reer R, Braumann KM. Video raster stereography back shape reconstruction: a reliability study for sagittal, frontal, and transversal plane parameters. Eur Spine J 2015;24:262-269.

Shumway-Cook A, Woollacott MH. Motor control: translating research into clinical practice. 3rd ed. Philadelphia (PA): Lippincott Williams \& Wilkins; 2007.

Yi YS, Yoo SK, Lee DG, Park DS. Reliability and validity of rasterstereography measurement for spinal alignment in healthy subjects. Phys Ther Rehabil Sci 2016;5:22-28. 